\title{
Efectos de la radiación ultravioleta en asfaltos colombianos
}

\author{
Aging effects by ultra violet radiation on Colombian asphalts \\ Ultravioleta efeitos de radiação em asfaltos colombianos
}

Recibido: febrero de 2012

Aprobado: mayo de 2012
Joyce M. Rojas*

Heriberto Amado**

Wilmar D. Fernández ${ }^{* * *}$

Freddy A. Reyes****

\section{Resumen}

Esta investigación tuvo como objetivo el envejecimiento a corto y largo plazo de dos asfaltos colombianos (60/70 y 80/100 de Barrancabermeja) a través de ensayos como Horno Rotatorio de Película Delgada (RTFO, por sus siglas en inglés) y Envejecimiento Acelerado de Vasija a Presión (PAV, por sus siglas en inglés), y un ensayo adicional de RTFOT más radiación Ultra Violeta UV a dos periodos distintos de exposición. Las propiedades reológicas y físicas de estos ligantes fueron analizadas en todas sus etapas mediante ensayos de penetración, ductilidad, punto de ablandamiento, viscosímetro rotacional y Reómetro de Corte Dinámico (DSR, por sus siglas en inglés). Los resultados muestran que tanto para la caracterización física, como para la caracterización reológica, el comportamiento de las muestras de los ligantes tratados con RTFOT y PAV es similar a las muestras tratadas con RTFOT y radiación UV por un periodo de 100 horas.

Palabras clave: envejecimiento de asfaltos, envejecimiento de corto plazo y largo plazo, RTFO, PAV, Radiación Ultra Violeta.

\begin{abstract}
The aim of this research was to observe the long and short term aging of two Colombian asphalts (60/70 and 80/100 from Barrancabermeja) through Rolling Thin Film Oven Test (RTFOT), RTFOT plus Ultra Violet radiation (UV) and accelerate aging by Pressure Aging Vessel (PAV) tests during two different exposure periods. Physical and Rheological properties such as penetration, ductility, softening point, viscosity with Brookfield and DSR were analyzed in all the stages. Results from both rheological and physical characterization show no variation between samples analyzed by RTFOT and PAV in comparison with the ones evaluated with RTFOT plus UV radiation during 100 hours periods.
\end{abstract}

Keywords: Asphalt aging, short term aging, long term aging, RTFO, PAV, Ultra violet radiation

\footnotetext{
Magister en ingeniería civil, grupo CECATA, Pontificia Universidad Javeriana ingjoycer@hotmail.co.

** Magister en Ingeniería Civil, grupo CECATA, Pontificia Universidad Javeriana, hamado@javeriana.edu.co

*** Profesor Asociado Universidad Distrital grupo TOPOVIAL. Candidato PhD. Pontificia Universidad Javeriana wfernandez@udistrital.edu.co.

***** Profesor Titular, grupo de Investigación CECATA Pontificia Universidad Javeriana. Fredy.reyes@javeriana.edu.co
} 


\section{Resumo}

Este estudo teve como objetivo o envelhecimento curto e longo prazo, de 2 de asfalto Colômbia (60/70 e 80/100 de Barrancabermeja) através de ensaios como forno de filme fino rotativo (RTFO por sua sigla em Inglês) e navio Acelerado pressão (PAV por sua sigla em Inglês) e uma prova adicional de RTFOT Ultra Violeta mais radiação UV para dois períodos diferentes de exposição, as propriedades físicas e reológicas desses ligantes foram testados em cada etapa por meio de testes e penetração, ductilidade ponto, amolecimento viscosímetro, rotação e reômetro de cisalhamento dinâmico (DSR por sua sigla em Inglês). Os resultados mostram que tanto a caracterização física e para o comportamento reológico caracterização das amostras tratadas com ligantes RTFOT e PAV é semelhante ao RTFOT amostras tratadas e as radiações UV durante um período de 100 horas.

Palavras-chave: envelhecimento do asfalto, o envelhecimento de curto prazo, o envelhecimento a longo prazo, RTFO, PAV, a radiação ultra-violeta.

\section{Introducción}

El asfalto como material presenta gran importancia para el desarrollo de nuestra economía, pues se constituye como parte fundamental de los materiales compuestos utilizados en diversas capas de las estructuras de los pavimentos. Estas vías tradicionalmente se han venido dimensionando bajo métodos empíricos que provienen de bases de datos de innumerables ensayos, que no investigan a fondo la relación de los propiedades de los materiales empleados y los factores a los que son expuestos en servicio. Sin embargo, en la actualidad se tiene un creciente empleo de los métodos racionales que consideran de alguna manera las propiedades medidas de los materiales que son empleados en la construcción de las vías y la forma en que estos se afectan por su puesta en servicio.

No obstante lo comentado, aun no se tiene en cuenta de una manera directa el envejecimiento que experimenta el asfalto, entendiendo dicho envejecimiento como las afectaciones que son generadas en los materiales asfálticos del pavimento durante su etapa de servicio, la exposición al medio ambiente, cargas repetitivas y los rayos ultravioleta por la exposición diaria a la radiación solar. Por lo tanto, para que este factor que interactúa a diario con los materiales asfálticos sea incluido de alguna manera formal en un proceso de diseño de pavimentos, primero deberán establecerse claramente las afectaciones que se producen por la radiación solar y en qué proporción se afectan las propiedades mecánicas de los ligantes asfálticos.

\section{Antecedentes}

Las investigaciones sobre las afectaciones experimentadas por el asfalto en sus propiedades mecánicas debido al envejecimiento se remontan a Vallerga (1957) y Hveem (1963). En el trabajo de Vallerga (1957) se tiene como conclusión por resaltar que la radiación ultravioleta se considera más dañina que la radiación infrarroja en ensayos realizados a películas de ligantes bituminosos de 3.125 $\mathrm{mm}$ de espesor. De la misma forma, y como antecedentes generales Traxler (1963) y Edler (1985), encontraron en sus investigaciones que el envejecimiento estaba relacionado con el espesor de película utilizada para las pruebas llevadas a cabo sobre el material.

Petersen (1984) describe diferentes causas del endurecimiento del asfalto, dentro de las cuales Bell (1987) resalta la pérdida de aceites por volatilización, el cambio en su estructura debido a la oxidación y el endurecimiento estérico; de hecho, estas son las más aceptadas en la literatura actual. 
Continuando con el trabajo de Bell (1987), realizado dentro del programa SHRP, este autor realizó una gran recopilación de información existente a esa fecha y a su vez estableció de manera formal recomendaciones sobre los ensayos que se deberían instaurar para simular el envejecimiento en ligantes asfálticos y de manera separada para el envejecimiento a corto y largo plazo. La primera etapa abarca el proceso de mezclado en planta, transporte y colocación de la carpeta en la vía y el segundo al periodo de servicio a partir de la instalación. Para simular los procesos mencionados, Bell recomendó utilizar el calor y el aire a presión como elementos fundamentales para el tratamiento de corto plazo, y el calor, la presión y la exposición ultravioleta para el tratamiento a largo plazo.

Posteriormente, y relacionado con la exposición a la radiación ultravioleta del material asfáltico, Bocci y Cerni (2000) encontraron en sus investigaciones que la radiación ultravioleta afecta de forma diferente al ligante, dependiendo del origen de la muestra; es decir, el proceso se presenta con variaciones dependientes de los componentes particulares de cada asfalto. Así las cosas, la fuente del material es determinante en su desempeño, ya que cada asfalto es diferente aun cuando provenga de fuentes muy cercanas geográficamente.

Afanasieva y Cifuentes (2002) presentaron un trabajo de investigación relacionando directamente la radiación solar en el proceso de envejecimiento en los asfaltos colombianos y encontraron que los rayos ultravioleta B son los que causan mayor afectación a los ligantes asfálticos. En el 2003, Suarez realizó una investigación en la cual caracterizó mediante DSR muestras de asfaltos colombianos provenientes de las fuentes de Apiay y Barrancabermeja en estado natural y luego de ser sometidos a procedimientos de simulación de envejecimiento a corto y largo plazo, mediante RTFOT y PAV. A partir de esto, encontró efectivamente que la variación de las propiedades se da a una tasa diferente entre los dos asfaltos colombianos y a su vez encontró módulos complejos más altos para el asfalto de la fuente Apiay.

Martínez y Caicedo en el 2005 realizaron una investigación para revisar los efectos de radiación ultravioleta en el envejecimiento de ligantes y mezclas asfálticas en asfalto proveniente de la fuente de Barrancabermeja. En este trabajo realizaron mediciones de módulo complejo y ángulo de fase al asfalto natural, al asfalto luego de RTFOT y UV, y al asfalto luego de RTFOT, PAV y UV. De esta manera, establecieron que la afectación de los rayos ultravioleta se encontraba relacionada con el espesor de la película utilizada $(2 \mathrm{~mm}$ y $1 \mathrm{~mm})$.

En 2008, Reyes y Camacho enfocaron el efecto de los rayos ultravioleta en las propiedades de una mezcla asfáltica partiendo del mismo principio de la caracterización del asfalto original y posteriormente envejeciéndolo mediante rayos ultravioleta. En este mismo año, Wuang realizó un proceso de envejecimiento por exposición natural a diferentes periodos de tiempo sobre un asfalto de penetración 65 , y encontró que por el proceso de oxidación sufrido el material se rigidiza; aspecto representado en el aumento del módulo complejo del material relacionado proporcionalmente con el tiempo de exposición al ambiente presentado. En 2009, Wu, et al, encontraron que la estructura del asfalto se modificaba paulatinamente con el envejecimiento sufrido, representado en la asociación progresiva de asfaltenos. El envejecimiento producido al ligante se realizó mediante RTFOT y PAV.

\section{Materiales y métodos}

Los asfaltos utilizados son los correspondientes a penetraciones $60 / 70$ y 80/100 provenientes de la planta de producción de Barrancabermeja Colombia. Estos asfaltos se caracterizaron en su estado original con los ensayos normalizados (ver Tabla 1), y sometidos a tratamientos de envejecimiento a partir de las normas mostradas en la Tabla 2.

\begin{tabular}{ll}
\hline ENSAYO & NORMA \\
\hline Penetración & I.N.V.-E 706 (ASTM D 5-97) \\
Viscosidad & I.N.V.-E 716 (ASTM D 4402) \\
Punto de ablandamiento & I.N.V.-E 712 (ASTM D 36-95) \\
Ductilidad & I.N.V.-E 702 (ASTM D 113-99) \\
\hline Tabla 1. Caracterización física \\
\hline
\end{tabular}




\begin{tabular}{ll}
\hline ENSAYO & NORMA \\
\hline RTFOT & I.N.V.-E 720 (D 2872) \\
PAV & I.N.V.-E 751 (D 2872) \\
DSR & I.N.V.-E 750 (D7175) \\
UV & Sin Norma \\
\hline
\end{tabular}

Tabla 2. Tratamientos de Envejecimiento

El envejecimiento a corto plazo es el proceso en el cual se toma muestra de un ligante en estado natural, se somete a un envejecimiento mediante el ensayo RTFOT simulando el envejecimiento que sufre esta muestra en el proceso de mezcla y puesta en obra; mientras que en el envejecimiento a largo plazo el ligante es sometido a dos ensayos RTFOT mas PAV, simulando el envejecimiento que sufre el ligante al paso de siete años aproximadamente. Sin embargo, se hizo un procedimiento de envejecimiento adicional que consistió en el tratamiento de las muestras de ligante original mediante ensayo RTFOT, y el residuo de este procedimiento a su vez fue expuesto a rayos ultravioleta en periodos de tiempo definidos.
La medición del envejecimiento se realizó mediante el análisis de las propiedades reológicas y físicas del ligante. Las primeras se obtuvieron mediante ensayos convencionales como viscosímetro rotacional, DSR y las segundas a partir de ensayos de penetración, punto de ablandamiento y ductilidad. Para el análisis de los datos se tomó como envejecimiento de corto plazo el material sometido a radiación UV sin el paso previo por el RTFOT y el envejecimiento de largo plazo al material tratado RTFOT mas las horas de exposición a la radiación UV. En las Figura 1 y 2 se muestran las caracterizaciones realizadas para las muestras de asfalto original y las muestras envejecidas a corto plazo mediante RTFOT.

\section{Resultados}

Se continuó con el proceso de envejecer el ligante en estado natural mediante el ensayo de RTFOT con los parámetros tradicionales, como lo indica la norma y su respectiva caracterización; posteriormente, se continuó con los envejecimientos a largo plazo y sus respectivas caracterizaciones físicas, lo cual dio los resultados que se presentan a continuación.

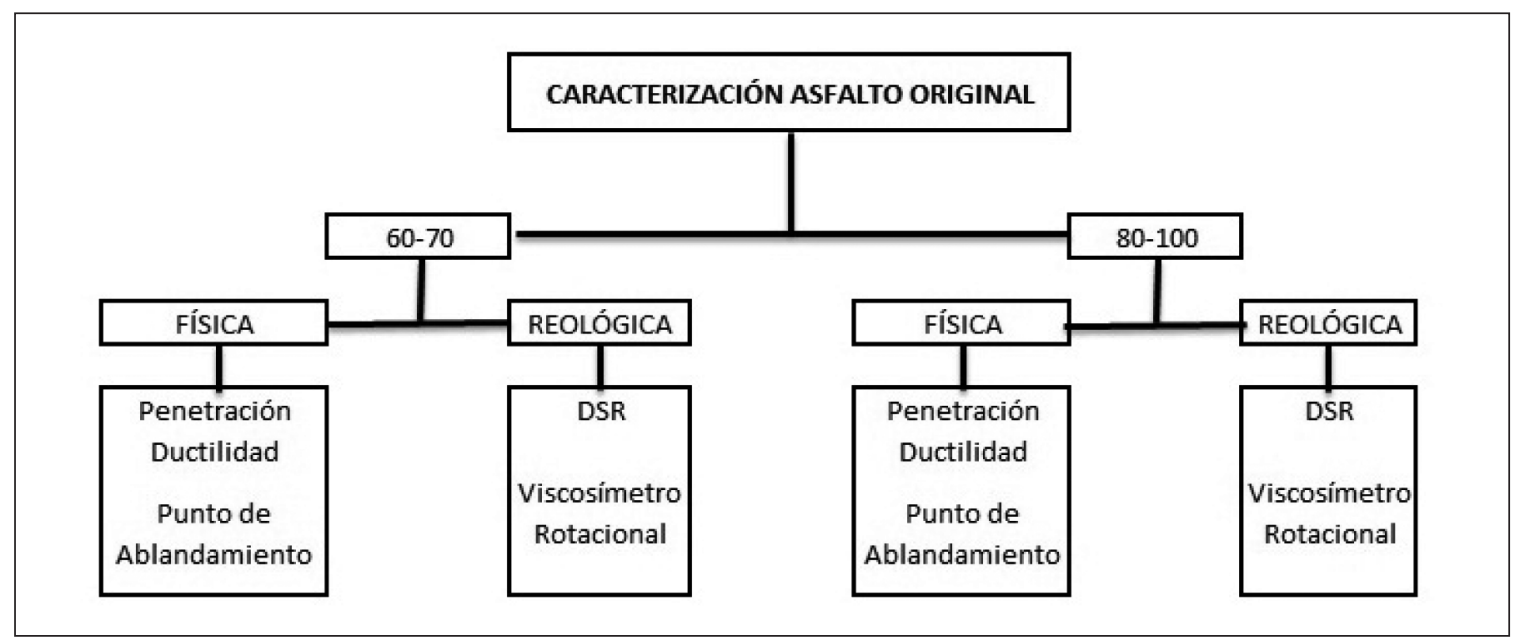

Figura 1. Caracterización asfalto original 


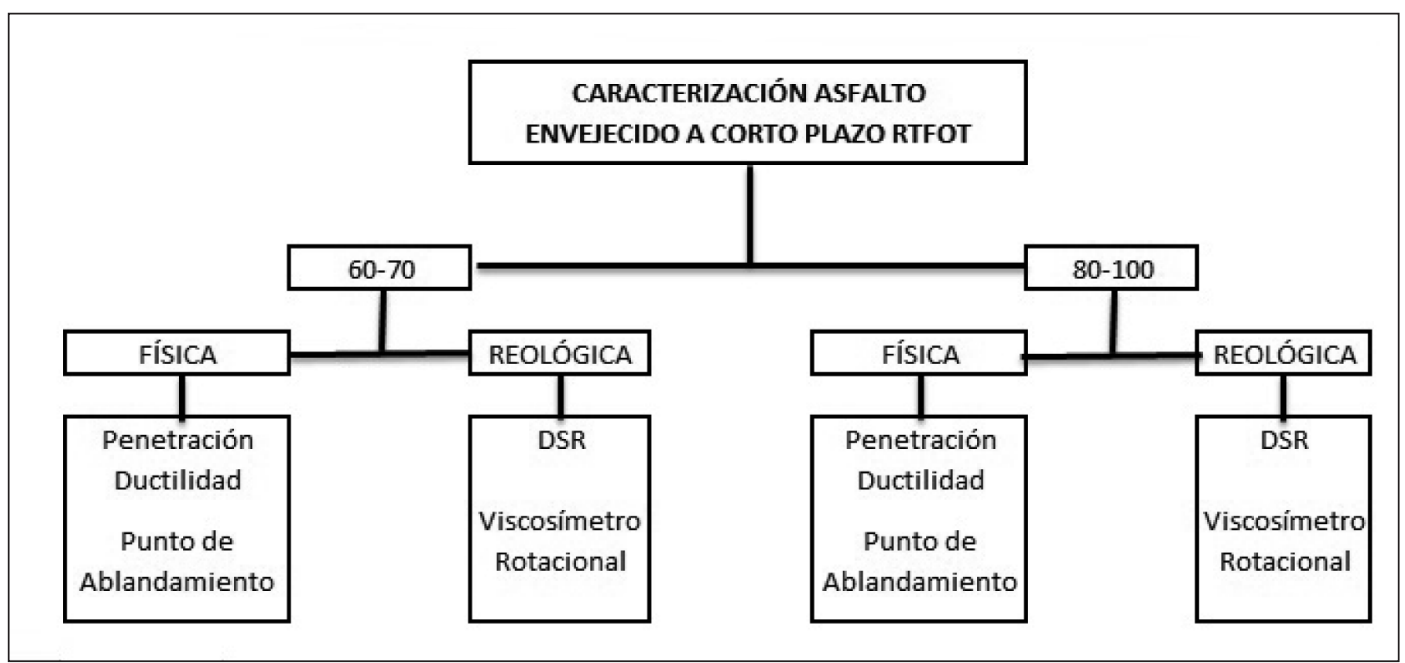

Figura 2. Caracterización asfalto envejecido a corto plazo

En la ductilidad comparada en el corto plazo, presentada en la Figura 3, los resultados muestran que las proporciones en la reducción de los resultados para este ensayo son símiles para los dos asfaltos tratados, y tienen además reducciones en los valores después del tratamiento RTFOT de $17 \%$ para el asfalto de penetración 60/70 y de 15\% para el asfalto de penetración $80 / 100$. El mayor porcentaje de reducción presentado para el asfalto de menor penetración puede entenderse si se considera que el procedimiento de envejecimiento a corto plazo afecta los componentes más livianos del ligante, que a su vez están presentes en menor proporción en el asfalto más rígido.

Como se muestra igualmente en la Figura 3, la ductilidad de largo plazo presenta un comportamiento descendente en el tratamiento con PAV; no obstante, y contrario a lo esperado, la ductilidad en los tratamientos con radiación UV presentan un comportamiento un aumento en la ductilidad. Esto demuestra la necesidad de profundizar en la revisión de este tipo de tratamiento complementando la caracterización con análisis químicos para verificar si se presenta una reducción,en el transcurso del proceso de irradiación, en mayor proporción de las fracciones pesadas del asfalto que, si bien rigidizan en conjunto la muestra, en el caso particular de la ductilidad hacen que la proporciones de asfaltenos presentes sea aún menor que el resto de componentes.
En cuanto al punto de ablandamiento medido para los dos ligantes caracterizados, puede considerarse que los resultados se presentan sin modificaciones al ser sometida la muestra al tratamiento de envejecimiento a corto plazo (ver Figura 4); hecho que permite deducir que el ensayo RTFOT no afecta la propiedad del asfalto de retener los agregados cuando el bitumen está presente en una mezcla asfáltica. Esta situación es concordante con la realidad, si se tiene en cuenta que una mezcla asfáltica recién colocada en condiciones normales no presenta problemas de desprendimiento de agregados, afectación que se va dando con el paso del tiempo en condiciones de servicio.

Continuando con la revisión de resultados, se observa en la Figura 4 cómo los resultados del ensayo de punto ablandamiento sobre muestras sometidas a procesos de envejecimiento de largo plazo presentan, a excepción del método con rayos ultravioleta por $50 \mathrm{~h}$ que permanece similar al ligante original, un leve aumento en la temperatura leída, lo que indica una rigidización de los asfaltos tratados.

Entrando en la revisión de los resultados del ensayo de penetración, se observa cómo en el tratamiento a corto plazo (ver Figura 5) el ligante, sin importar su penetración inicial, aumenta en proporciones similares la dureza, lo que se muestra como otro indicio de que el tratamiento con RTFOT afecta de forma similar los dos asfaltos tratados. 


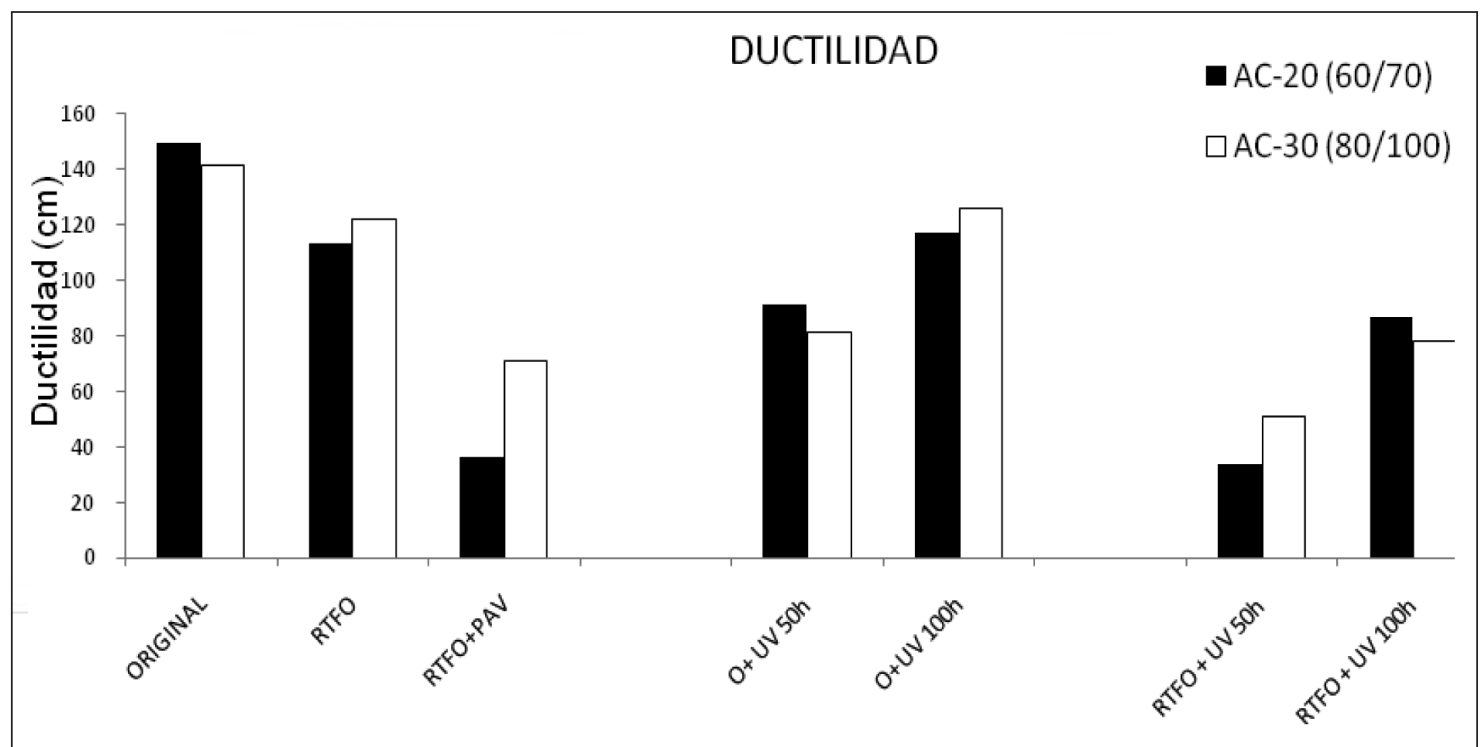

Figura 3. Ductilidad asfaltos $60 / 70$ y $80 / 100$

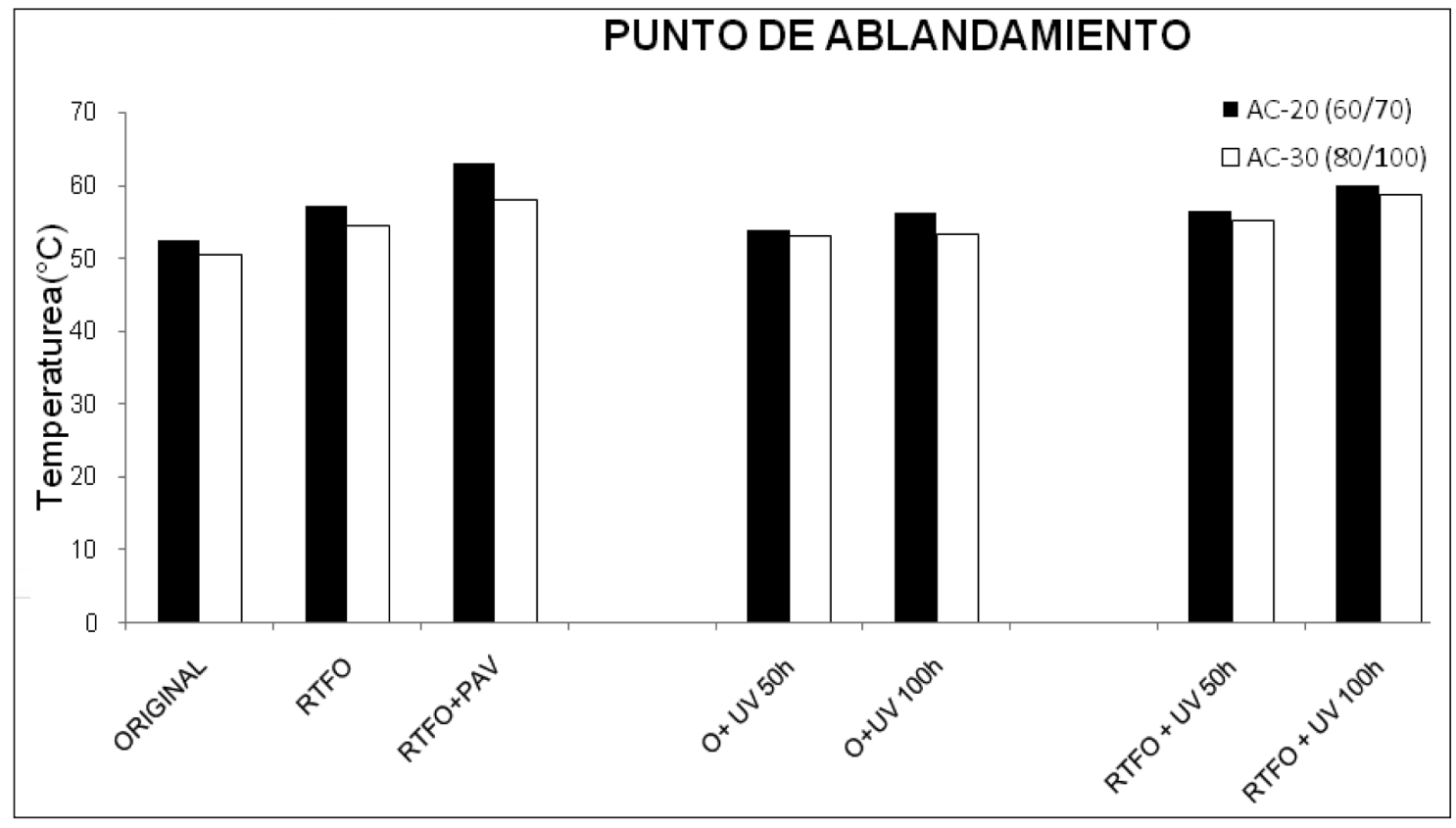

Figura 4. Punto de ablandamiento asfaltos $60 / 70$ y $80 / 100$

La rigidización del asfalto se hace marcadamente notoria cuando se revisan los resultados de penetración obtenidos sobre muestras sometidas a tratamientos de largo plazo, y cabe resaltar que el tratamiento con exposición a rayos UV presenta valores que no son consecuentes con el endurecimiento esperado, pues para las $50 \mathrm{~h}$ de exposición se tienen valores de penetración más altos que para el residuo tratado a corto plazo mediante RTFTO.
Una causa probable de esto es la dificultad para remoldear las muestras tratadas, tanto con PAV, como con irradiación con rayos UV para la ejecución del ensayo, pues en este procedimiento se presenta acumulación de aire en las probetas utilizadas para tal fin. Se aprecia también en la Figura 5 cómo para este ensayo los valores obtenidos mediante el tratamiento en PAV y UV de 100 horas presentan resultados comparables. 


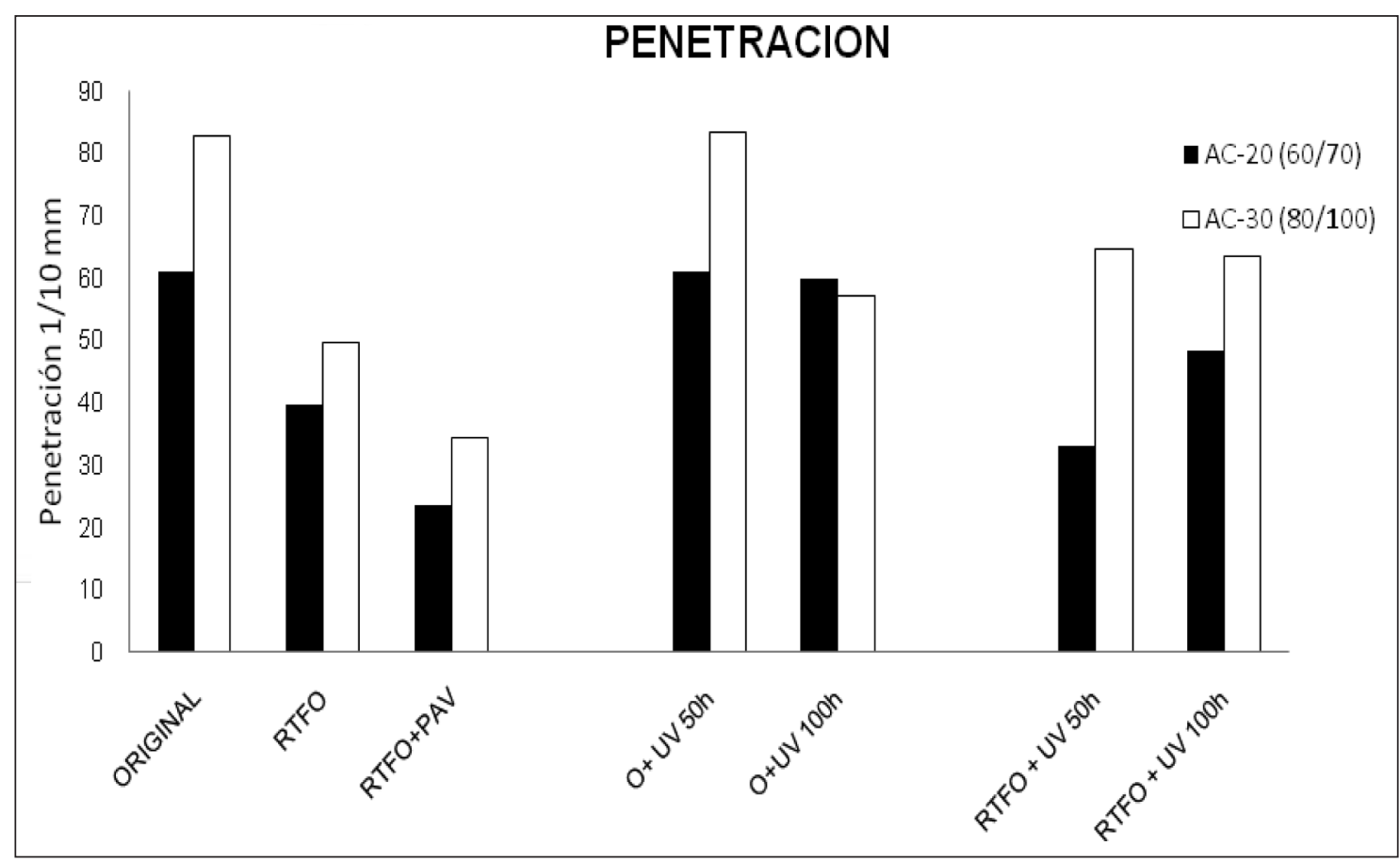

Figura 5. Penetración asfaltos $60 / 70$ y $80 / 100$

Los resultados de la caracterización reológica realizada con el DSR se enfocaron en la medición del ángulo de fase y el módulo complejo, presentados en las Figuras 6 y 7, respectivamente, para los ligantes originales tratados con RTFOT y con PAV. El ángulo de fase (ver Figura 6) se reduce en cuanto se aplica a la muestra el tratamiento de envejecimiento a corto plazo comparado a una misma temperatura. Con respecto al envejecimiento de largo plazo, de la misma manera hay una reducción en el ángulo de fase. A su vez, en la rigidez conservada se observa cómo se mantiene más rígido el asfalto original de menor penetración sin importar qué clase de tratamiento se aplique a la muestra.

En cuanto a la medición del módulo complejo (ver Figura 7), puede verse en los resultados que el tratamiento de envejecimiento a largo plazo induce un cambio de magnitud considerable en comparación con los valores obtenidos para las muestras de los ligantes en estado natural. Esta rigidización generada por el envejecimiento de largo plazo presenta resultados similares para los dos asfaltos tratados, lo que demuestra que en estado avanzado de servicio las diferencias existentes entre los dos asfaltos de diversa penetración inicial no son significativas; hecho que permite suponer que la composición química de los dos asfaltos es símil en este estado probablemente con valores iguales en sus fracciones.

Una representación de la rigidización continua de la mezcla a través de los tratamientos de envejecimiento es probada mediante el Cálculo del Factor de Ahuellamiento, según el programa de investigación SHRP; factor que es en sí mismo la componente viscosa del módulo complejo del material. Por lo tanto, y como se podría esperar de acuerdo con los resultados de las caracterizaciones físicas, este factor presenta un aumento en valor, lo cual indica la rigidez que adquiere el material de acuerdo con la disminución del ángulo de fase, prueba precisa del aumento de la fragilidad del material. El factor de ahuellamiento calculado para las muestras tartadas se presenta en la Figura 8. 


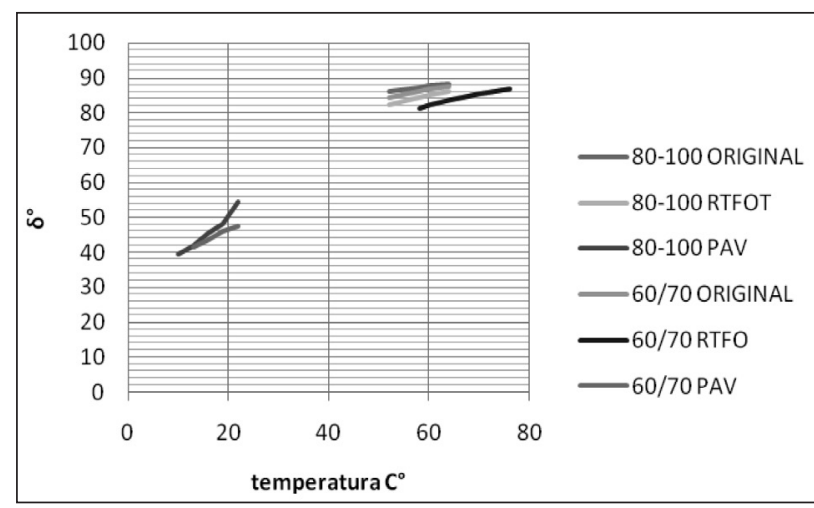

Figura 6. Comportamiento del ángulo de fase con la temperatura

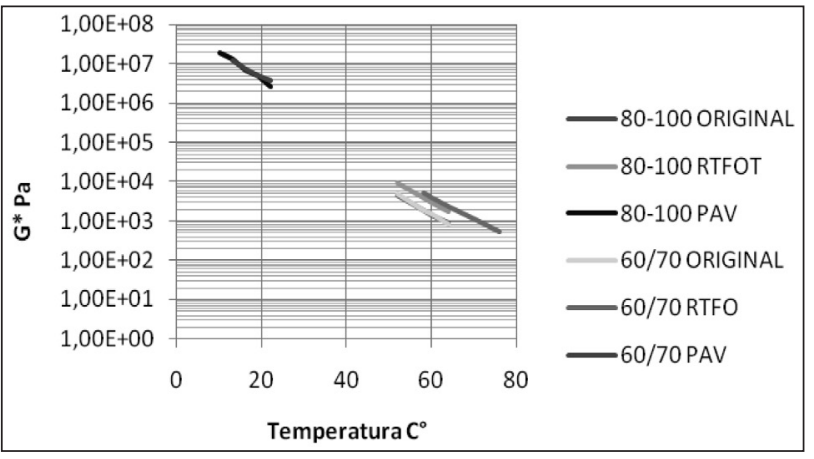

Figura 7. Evolución del módulo complejo con la temperatura

Para las muestras originales, envejecidas a corto plazo mediante RTFOT y a largo plazo mediante exposición por $200 \mathrm{~h}$ a rayos UV, se realizó la medición de su viscosidad a partir del viscosímetro rotacional, y se obtuvieron los valores que se presentan en las Figuras 9 y 10.

Como se observa en la Figura 9, la viscosidad medida en viscosímetro rotacional muestra igualmente un aumento en los valores obtenidos para los procesos de envejecimiento, lo que coincide con la tendencia de rigidización del ligante mostrada en las caracterizaciones presentadas en este documento, y de la misma manera coincidiendo en que el comportamiento que se presentó para el tratamiento a largo plazo con PAV presenta similutud con el presentado en la muestra tratada con envejecimiento a largo plazo mediante exposición a los rayos UV de 100 horas. Esta similitud entre mediciones de viscosidad es más importante en el ligante de penetración inicial 60/70 que en el ligante de penetración inicial 80/100 (ver Figura 10)

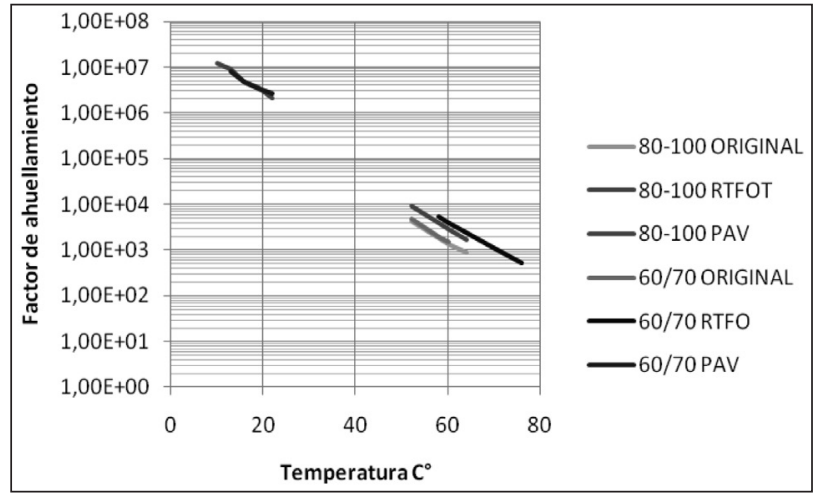

Figura 8. Factor de ahuellamiento

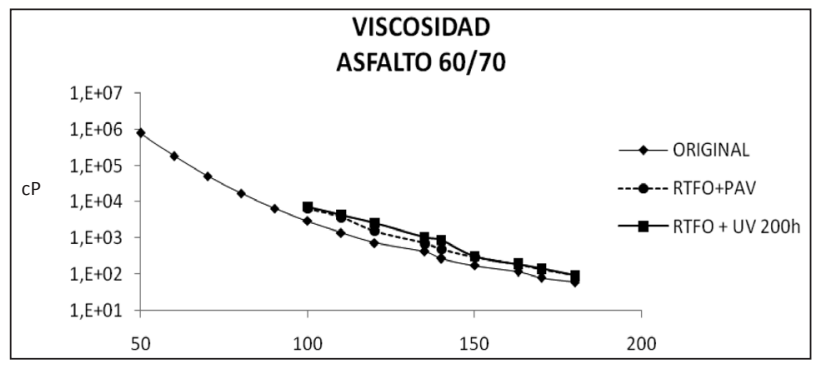

Figura 9. Comparación de viscosidad ligante 60/70 original y envejecido largo plazo*

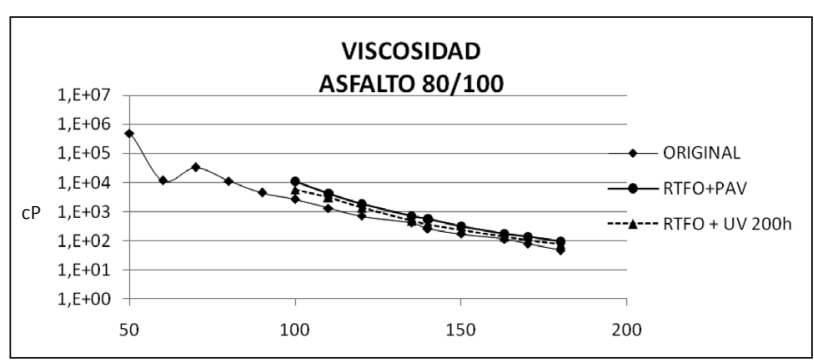

Figura 10. Comparación de viscosidad ligante 60/70 original y envejecido largo plazo*

* Los valores de viscosidades por debajo de $100{ }^{\circ} \mathrm{C}$ se encuentran en verificación para las muestras con tratamiento de envejecimiento a largo plazo.

\section{Conclusiones}

El tratamiento de envejecimiento a corto plazo mediante el ensayo RTFOT, desde el punto de vista de las caracterizaciones físicas, termina en cambios de poca magnitud en los resultados de 
punto de ablandamiento. No obstante, las magnitudes presentan una relación de cambio mayor para los resultados de ductilidad y de penetración. En cuanto a valores de módulo complejo y ángulo de fase, se presenta un aumento del primer parámetro y una reducción del segundo como muestra clara de la rigidización de los ligantes sometidos al tratamiento de envejecimiento de corto plazo; sin embargo, las variaciones medidas son bajas.

Para los tratamientos de envejecimiento a largo plazo los resultados de viscosidad y penetración el ensayo normativizado PAV puede ser comparado con un tratamiento de exposición a rayos UV de entre 100 horas y 200 horas, y es este último el que arroja más aproximación en los resultados de la caracterización reológica mediante el viscosímetro rotacional.

\section{Agradecimientos}

Agradecemos al laboratorio de Ingeniería Civil de la Pontificia Universidad Javeriana y al Químico Ernesto Villegas, de la Universidad de Costa Rica, por el apoyo y manejo de algunas muestras. Así mismo, a la empresa Concresol S. A. la cual apoyó en el suministro del material para las pruebas.

\section{Bibliografía}

Bell, C. (1989). Summary report on aging of asphalt-aggregate systems, Strategic Highway Research Program, National Research Council.

Bocci, M. y Cerni, G. (2000). The ultraviolet radiation in short-and long-term aging of bitumen.

Castillo, S. y Caicedo B.. (2005). Caracterización de asfaltos con tecnología 'superpave' y análisis de ahuellamiento[Trabajo de grado], Bogotá, Universidad de Los Andes, Maestria en Ingeniería Civil.

Khalid, H. y C. Walsh (2002). A new approach for the accelerated ageing of porous asphalt mixtures. Proceedings of the Institution of Civil Engineers, Transport. vol 153, pp 171-181.
Instituto Nacional de Vías, INVIAS. (1996). Medición de propiedades reológicas de los ligantes asfálticos mediante el reómetro de corte dinámico I.N.V. E- 750 - 07, octubre de 1996.

Instituto Nacional de Vías, INVIAS. (1996). Medición de envejecimiento acelerado de ligantes asfálticos utilizando cámara de envejecimiento a presión (pav) I.N.V. E- $751-07$, octubre de 1996.

Instituto Nacional de Vías, INVIAS. (1996). Ensayo en el horno de lámina asfáltica delgada en movimiento I.N.V. E - 720 - 07, octubre de 1996.

Lesueur, D. (2009). “The colloidal structure of bitumen: Consequences on the rheology and on the mechanisms of bitumen modification." En Advances in colloid and interface science vol. 145, núm. 1-2, pp. 42-82.

Martínez, G. y Caicedo, B. (2005). Efecto de la radiación ultravioleta en el envejecimiento de ligantes y mezclas asfálticas.[Trabajo de Grado], Bogotá, Universidad de Los Andes, Maestria en Ingeniería Civil.

Reyes, O y Camacho, J. (2008).”Efecto de la radiación ultravioleta en las propiedades mecanicas y dinámica de una mezcla asfáltica. En Ingeniería e Investigación vol. 28, núm. 3, pp. 22-27.

Wang, J., et al. (2008). "Effect of ageing on fatigue properties of asphalt". En Journal of Central South University of Technology, vol. 15, pp. 111-114.

Wu, S., L. Pang, et al. (2009). "Influence of aging on the evolution of structure, morphology and rheology of base and SBS modified bitumen". En Construction and Building Materials vol. 23, núm. 2, pp. 1005-1010.

Wu, S., G. Zhu, et al. (2009). "Laboratory research on thermal behavior and characterization of the ultraviolet aged asphalt binder". En Lournal of thermal analysis and calorimetry vol. 95, núm.2, pp. 595-599. 